\title{
Triple Collision Reaction of Deuterons as a Possible Explanation of Cold Nuclear Fusion
}

\author{
E. W. Becker \\ Strählerweg 18, D-7500 Karlsruhe 41
}

The publications by Fleischmann and Pons [1] and by Jones et al. [2] about an electrochemically induced fusion reaction of deuterium nuclei in a palladium cathode understandably have aroused worldwide attention. Particularly remarkable is the enthalpy production rate communicated by Fleischmann and Pons: In one of their experiments, parts of the cathode melted and evaporated, and other parts of the experimental setup were destroyed. The high enthalpy production rate cannot be explained in terms of the wellknown fusion reactions

$$
\begin{array}{r}
{ }^{2} \mathrm{D}+{ }^{2} \mathrm{D}={ }^{3} \mathrm{~T}(1.01 \mathrm{MeV})+ \\
{ }^{1} \mathrm{H}(3.02 \mathrm{MeV})
\end{array}
$$

or

$$
\begin{aligned}
{ }^{2} \mathrm{D}+{ }^{2} \mathrm{D}= & { }^{3} \mathrm{He}(0.82 \mathrm{MeV})+ \\
& \mathrm{n}(2.45 \mathrm{MeV})
\end{aligned}
$$

as the rates of neutron and tritium production observed are by far too low. According to a report published in the "Neue Zürcher Zeitung" of April 1, 1989, Fleischmann, in a lecture given at the Technical University of Lausanne on March 30, 1989, considered the formation of ${ }^{4} \mathrm{He}$ as an explanation of the discrepancy between the rates of enthalpy and neutron production. According to the report that explanation was received with scepticism by the physicists present in the audience. The scepticism is understandable, as the reaction

${ }^{2} \mathrm{D}+{ }^{2} \mathrm{D}={ }^{4} \mathrm{He}+\gamma$

proceeds with a cross-section of less than $10^{-7}$ barn [3] under customary conditions, while cross-sections in excess of $10^{-2}$ barn are attained in reactions (1) and (2). The large difference is due to the fact that in reactions (1) and (2) the two heavy daughter particles easily meet the conservation of momentum while reaction (3) is impeded because of the slow energy dissipation by $\gamma$-quanta.

When looking for a nuclear reaction not observed as yet, which could explain the incongruity between enthalpy and neutron production, one has to consider the uncommonly high density of the deuterons in electrochemically charged palladium which, according to Fleischmann and Pons [1], may result in "deuterium clusters" forming. These clusters, which in all likelihood include $\left(\mathrm{d}_{3} \mathrm{e}_{2}\right)^{+}$configurations, could release a triple-collision reaction:

$$
\begin{aligned}
& { }^{2} \mathrm{D}+{ }^{2} \mathrm{D}+{ }^{2} \mathrm{D}={ }^{6} \mathrm{Li}^{*} \\
& ={ }^{4} \mathrm{He}(\approx 8 \mathrm{MeV})+{ }^{2} \mathrm{D}(\approx 16 \mathrm{MeV})
\end{aligned}
$$

Since this reaction which proceeds via an excited ${ }^{6} \mathrm{Li}^{*}$ configuration produces two heavy daughter particles the law of conservation of momentum is easily observed.

To explain Fleischmann's results, the effectiveness of the hypothetical reaction (4) has to be several orders of magnitude higher than that of the wellknown reactions (1) and (2). Such higher effectiveness could not only be due to more favorable kinematic conditions, but also to the release of the fast deuteron in reaction (4) facilitating the initiation of further fusion reactions.

If Fleischmann's results can be confirmed with respect to the ratio of enthalpy and neutron production, and if the explanation given above is correct, cold fusion would allow nuclear power to be produced largely free from problems of radioactivity. This statement would have to be qualified slightly if the excited ${ }^{6} \mathrm{Li}$ nucleus would decay into ${ }^{3} \mathrm{~T}$ and ${ }^{3} \mathrm{He}$ instead of ${ }^{4} \mathrm{He}$ and ${ }^{2} \mathrm{D}$, which is also conceivable. Studies are under way in Karlsruhe to detect the He isotopes.

I thank G. Falk and G. Schatz for stimulating comments.

Received April 18, 1989

1. Fleischmann, M., Pons, S.: Electroanal. Chem. (submitted)

2. Jones, S. E., Palmer, E. P., Czirr, J. B., Decker, D. L., Jensen, G. L., Thorne, J. M., Taylor, S. F.: Nature (submitted)

3. Zurmühle, R. W., Stephens, W. E., Staub, H. H.: Phys. Rev. 132, 751 (1963)

Naturwissenschaften 76, 214-215 (1989) (C) Springer-Verlag 1989

\section{Debye Length and Surface Tension in Nuclei}

\author{
H. Hora* \\ Department of Theoretical Physics, University of New South Wales, \\ Kensington 2033, Australia
}

The recently achieved explanation of surface tension in metals by plasma double layers [1] is extended to nuclei in

\footnotetext{
* At present Visiting International Scholar, Department of Physics, University of Missouri, St. Louis, Missouri 63121, USA.
}

order to explain the measured [2] decay length of the charge distribution in the surface of nuclei as a kind of Debye length and to derive then the wellknown measured [3] nuclear surface energy.

The appearance of double layers and high electric fields in the surface as well as in the interior of laser-produced plasmas [4] was the starting point for deriving surface tension in plasmas [5]. Electrons leaving the surface faster than ions at the same energy produce an electric double layer. The electricfield energy per area results in surface tension which damps surface waves of short wavelength and acts against Rayleigh-Taylor instabilities [5]. In a similar way there is the question as to how the degenerate electron gas between the ions of the rigid lattice in a metal pushes outside, produces a double layer, and results in surface tension. 